\title{
Weitere Mittheilungen über Kern- und Zelltheilungen in der Milz; zugleich ein Beitrag zur Kenntniss der von der typischen Mitose abweichenden Kerntheilungsvorgänge. \\ Von
}

Prof. Dr. Inl. Armold in Heidelberg.

Hierzu Tafel XXV, XXVI, XXVII.

Als die wesentlichste Aufgabe der Erforschung karyomitotischer Vorgänge wurde bisher der Nachweis der Architektur und Struktur der Kerne in den einzelnen Phasen der mitotischen Zweitheilung, sowie des Vorkommens dieser unter normalen und pathologischen Verhältnissen betrachtet.

$\mathrm{Ob}$ Abweichungen von diesem Typus der Zweitheilung und Uebergangsformen zwischen der mitotischen und amitotischen Kerntheilung, deren Existenzberechtigung von den Meisten allerdings in Frage gestellt wird, bestehen, darüber finden sich nur vereinzelte Angaben.

Dic pluripolaren Mitosen, welche zuerst in das Gebiet der Phantasie verwiesen, später als Artefacte aufgefasst wurden, bezeichnet man jetzt als "anomale" oder "patbologische" Erscheinungen. Nachdem nachgewiesen ist ${ }^{1}$ ), dass diese vielpoligen Figuren bezüglich ihrer Architektur und Struktur nur scheinbare Abweichungen, in Wirklichkeit aber die typische Anordnung darbieten, welche durch die gleichzeitige Theilung in mehrere Kerne vorgezeichnet ist, kann eine solche Benennung nicht mehr als zutreffend gelten. Wollte man aber dieselben als "anomale" oder "pathologische" Formen deshalb deuten, weil sie bis jetzt bauptsächlich unter pathologischen Bedingungen beobachtet wurden, so genügt der Hinweis auf deren Vorkommen im

1) Vergleiche Schottländer, über Kerntheilungsvorgänge in dem Endothel der arteficiell entzündeten Hornhaut, dieses Archiv Bd. XXI. S. 426; daselbst findet sich die ganze auf diese Frage bezügliche Literatur. 
normalen Knochenmark, um die Berechtigung eincr solchen Schlussfolgerung zu widerlegen. Ueberhaupt wäre es voreilig, aus den spärlichen Mittheilungen über pluripolare Mitosen anf ibr seltenes Vorkommen zu schliessen; es ist eben diesen Fornen die Beachtung, welche sie meines Erachtens verdienen, bis jetzt noch nicht zu Theil geworden.

Ein anderes Bewandtniss hat es mit den wirklichen $A b$ weichungen der Architektur und Struktur, wie sic bei der mitotischen Zweitheilung in den versehiedenen Phasen vorkommen und zum Theil zuerst von $\mathrm{Carnoy}{ }^{1}$ ), später von Flemmin $g^{2}$ ) eingehend beschrieben und von dem letztgenannten Autor mit dem Namen der homöotypischen und heterotypischen Mitosen belegt worden sind.

Die ver\%̈grerten Umlagerungen der chromatischen Fïden, wie sie namentlich im Stadium der äquatorialen Umorduung, ebenso die verspäteten Abschnürungen, welehe in späteren Stadien getroffen werden, sind wohl richtiger als $A$ berrationen ${ }^{3}$ ), nicht als Abweichungen von der typischen Mitose aufzufassen.

Wiederum eine andere Stellung gebiihrt den von mir als indirekte Fragmentirung bezeichneten Theilungrvorgiingen, welche nit der echten Mitose die Zunahme der chromatischen Sub$\operatorname{stan} z$ gemein haben, von dieser aber durch die Anordnungr namentlich in den späteren Phasen sich unterscheiden.

Meine früheren Mittheilungen über atypische Mitosen iiberbaupt, indirekte Fragmentirung insbesondere sind vielfach missverstanden und nur von Wenigen einer sachlichen Controle unterworfen worden. Nachdem jetzt feststeht, dass derartige Abweichungen und Aberrationen von der typischen Mitose vorkommen, findet der nachfolgende Bericht vielleicht geneigteres Gehör und zur objektiven Nachuntersuchung willigere Augen. - Durch die bisherigen Erfahrungen nicht gebessert, trage ich mich mit der Erwartung, dass das Untersuchungsobjekt, welches ich zu diesem Behufe in Vorschlag bringe, wegen seiner Vorziuge auch bei Anderen Beifall findet. Es ist die Milz der weissen Maus wegen

1) Carnoy, les cytodiérèse chez les Arthropodes. 1885.

2) Flemming, neue Beiträge zur Kenntniss der Zelle, dieses Archiv Bd. XXIV, H. 3, 1887.

3) Schottländer 1. c. 
ibrer Kleinheit sowohl, als auch wegen des Reichthums an atypischen Kerntheilungsfiguren sehr zu empfehlen. Man kann dieselbe ohne weitere Vorbereitungen, nachdem man sie möglichst schnell herausgeschnitten und balbirt hat, in die bei solchen Untersuchungen gebräuchlichen Reagentien einlegen. Die Zahl derartiger Kerntheilungsfiguren ist immer eine so beträchtliche, dass sie auf jedem Schnitt nicht nur vereinzelt, sondern in grösserer Auswahl getroffen werden: ein Verhältniss, welches darauf hinweist, dass wir es in diesen Formen nicht mit zufälligen und nebensiichlichen Vorkommnissen zu thun haben, dass sie vielmehr bei der Theilung lymphoider Zellen eine hervorragende Rolle spielen. - Vielleicht kann an diesem Objekte bei gemeinsamer vorurtheilsfreier Arbeit niclt nur die Frage uber die Existenz derartiger Kerntheilungsfiguren, sondern auch über deren Beziehung einerseits zur echten mitotischen, andererseits zur amitotischen Kerntheilung, sowie die Bedeutung beider für die Kerntheilungsvorgänge an den lymphoiden Zellen, wenn nicht gelöst, so doch wesentlich gefördert werden. Dazu einen kleinen Beitrag zu liefern, soll in den nachfolgenden Zeilen der Versuch gemacht werden.

Einem früher ausgesprochenen Grundsatz getrea habe ich auch bei diesen Untersuchungen die wichtigsten Methoden angewendet. Einen besonders ausgedehnten Gebrauch machte ich von Chrom-Osmium-Essigsïure (schwaches Fle m ming'sches Gemiscb), Chrom-Ameisensïure (Rabl), Chrom-Essigsäure ${ }^{1}$ ), PlatinchloridChrom-Essigsäiure $\left.{ }^{2}\right)$ und endlich reines Platinchlorid $(1 / 3 \%)$. Einen sebr wesentlichen Unterschied habe ich zwischen der Wirkung der anfgeführten Reagentien, von dem letzterwähnten abgesehen, nicht wahrgenommen. Die Chrom-Essigsäure bietet den grossen Vortheil, dass sie rascher und vollständiger die Gewebe durchtränkt als die anderen Chromgemenge, die Chrom-Osmium-Essigsäure insbesondere. Die Objekte brauchen nicht länger wie 24 Stunden in circa 10-15 ccm dieser Conservirungsflüssigkeiten liegen zu bleiben und werden dann mit Alkohol im Dunklen in der ibblichen Weise behandelt. Die in reinen $(1 / 3 \%)$ Platinchloridlösungen gehärteten Präparate zeigen mehr das Verhalten, wie die in Alkohol conservirten, deren Studium zur Controle un-

1) Chromsäure 0,3, Essigsäure 0,5 anf 100 Wasser.

2) Platinchlorid 0,45, Chromsäure 0,1, concentrirte Essigsäure 9,4 auf 100 Wasser. 
Jul. Arnold:

entbehrlich ist. Sehr gute Resultate erhält man auch bei der Härtung in Spiritus, dessen Concentration von $25 \%$ an bis zn $96 \%$ innerhalb $36-48$ Stunden gesteigert wird. - Dass bei der Einhaltung derselben Härtungsmethode doch verschiedene Effekte erzielt werden, ist eine Erfahrung, welche ich auch bei diesen Untersuchungen zu machen Gelegenbeit hatte. Zum Färben der Präparate verwendete ich Hämatoxylin (schwache Lösungen) und Safranin (mit Anilinöl). Sehr empfehlenswerth finde ich die von Bizzozero angegebene Modifikation der Gram'schen Methode (Chromsüure 0,1\% statt Jod zur Entfärbung). Da sehr feine Schnitte erforderlich sind, ist die Einbettung in Paraffin der Durchtränkung mit Celloidin vorzuziehen; doch erhält man auch mittelst der letzteren Methode sebr brauchbare Prïparate. Von stirkeren Systemen standen mir zu Gebot Zeis $1 / 12$ und $1 / 1$. homog. Immers. und apochromat. homog. Immers. 1,30-20 mm.

Bei der Darstellung der Befunde will ich so vorgehen, dass ich zunächst die verschiedenen Formen, wie sie in der Milz vorkommen, beschreibe und dann erst prïfe, in welcher Bezichung sie zu den bekannten Typen der Kerntheilung stehen.

Man trifft in der Milz der weissen Maus lymphoide Zellen, welche in Bezug auf Grösse und Form des Zellleihes und der Kerne, sowie betreffs Architektur und Strnktur der letzteren die grössten Abweichungen darbieten. Von den kleinen Zellen mit kaum nachweisbarem Protoplasma bis zu den ganz grossen finden sich zahlreiche Uebergänge. Noch variabler ist die Architektur und Struktur der Kerne sowohl bei den grossen als bei den kleinen Zellen. Es würde vielleicht möglich sein, für gewisse Gruppen von Zellen gemeinsame morphologische Eigenschaften nachzuweisen. So lange aber nicht festgestellt ist, dass diesen eine verschiedene Herkunft oder eine differente funktionelle $\mathrm{Be}$ deutung zukommt, wäre eine derartige Trennung doch nur von zweifelhaftem Werthe. Dazu kommt, dass die wechselnde Architektur nnd Struktur der Kerne, wenn nicht ausschliesslicb, so doch vorwiegend zu den Theilungsvorgängen in Beziehung gebracht werden muss. Das sind die Grïnde, warum es sich empfiehlt, zunächst nur zwei Formen von lymphoiden Zellen zu unterscheiden: die kleineren und die ganz grossen. Dass zwischen diesen alle möglichen Abstufungen und Uebergäinge getroffen werden, will ich nicht unterlassen noch einmal zu betonen. 
Die Kerne der kleinen Zellen sind im Ruhezustand hell; sie führen gewöhnlich mehrere Kernkörperchen von rundlicher Form oder mehr eckige Gebilde, welche intensiv sich mit Farbstoffen tingiren, ausserdem aber helle Fäden, iaber deren Anordnung, Verlaufweise und gegenseitige Beziehung bestimmte Angaben unmöglich sind. Die Kerne insbesondere der kleinsten Zellen baben zuweilen einen eigenthümlichen Glanz und färben sich so dunkel, dass man nur bei intensiver Durchleuchtung oder nach der Behandlung mit angesäuertem Alkohol dunkle Körnchen und Fädchen in ibnen erkennen kann. Ob diese Erscheinnng einer eigenartigen Struktur oder einem Contraktionszustand der Kerne entspricht oder ob die Kerne als in Vorbereitung zur Theilung begriffen angeseben werden mïssen, diese Fragen habe ich schon an einer anderen Stelle erörtert ${ }^{1}$ ).

In der ersten Phase der Theilung nehmen die rundlichen und eckigen intensiv sich färbenden Gebilde an Zahl und Umfang zu, die zwischen ilnen verlaufenden Fädchen treten zum Theil dentlicher hervor (Tafel XXV Fig. 1 bis 11) und werden dicker, glänzender und dunkler. In der Kernmembran, welche zuweilen schon in diesem Stadium die Eigenschaften einer chromatischen angenommen hat, werden gewöhnlich in gleichen Abständen kleine glänzende Körner kenntlich, welche zu den Fäden in Beziehung zu stehen, manchmal Querschnitte derselben zu sein scheinen (Tafel XXV Fig. 2, 3, 6 u. 8). - Diese eben erwähnten Strukturveränderungen vollziehen sich an den Kernen in ungleichartiger Weise; entweder gleichzeitig in der Ausdehnung des ganzen Kerns, gewöhnlich an der einen oder anderen Stelle früher oder an mehreren Stellen zu derselben Zeit. Die dunklen in den Kernen entstehenden Figuren sind bald ringförmig, bald erscheinen sie als einfache, mehrfache oder verzweigte Balken oder sonstwie gestaltete Gebilde (Tafel XXV Fig. 1-15). Einzelne dieser Balken sind so dick, dass sie überhaupt nicht wie aus einzelnen Fäden, sondern aus einer grösseren Zahl solcher zusammengesetzt erscheinen (Fig. 4 u. 5). Die chromatische Kernmembran ist in diesem Stadium noch sehr deutlich. In derselben Weise gestaltet sich die Zunahme der chromatischen Substanz in den Kernen, welche

1) J. Arnold, über Theilungsvorgänge an den Wanderzellen, dieses Archiv Bd. 30, H. 2, S. 250. 
in die Länge gezogen sind oder als gewundene oder aufgerollte Bïnder sicb darstellen (Tafel XXV Fig. 19-23).

In der nächsten Phase werden die Kerne nehr gleichmäissig von Füden durchsetat, welche aber in ihrer Anordnung glcichfalls beträchtliche Verschiedenheiten darbieten. Die Fäden sind bald sehr fein, bald dicker oder aber es finden sich mehr bandartige Gebilde, in deren Innerem sich zuweilen mehrere Fiiden nachweisen lassen. Die Aufstellung der Fidden ist zuweilen eine radiüre, haiufiger erbält man den Eindruck, als ob dieselben in net/formiger Verbindung stainden; doch gelingt es an den kleinen Kemen nicht, darüber zu entscheiden. Die Fïrbung der Fäden erscheint bald als eine gleichmissige, bald sind einzelne Abschnitte derselben heller, andere dunkler tingirt; so werlen z. B. in dem Kern, welcher in Fig. 15 abgebildet ist, die heller gefurbten Fiden durch dunkle Körner in ilrem Verlauf unterbrochen. Viele Kerne bieten in diesem Stadium bald nur eine schwache, bald eine stärkere, an verschiedenen Stellen gleichmässige oder ungrleichmäissige diffuse Färbung dar (Fig. 17, 18, 22 u. 23). An Präparaten, welche mit saurem Alkohol behandelt wurden, pflegt diese eine weniger intensive zu sein; es liisst sich desshalb an solchen der Nachweis führen, dass auch solche Kerne chromatische Fïden in mehr oder weniger grosser Zahl enthalten und dass die Kerne nicht homogen sind, wie man nach einer flichtigen Untersuchung vermuthen möchte. Die in dic Lïnge gezogenen, gelappten, gewundenen und anfgerollten Kerne zeigen im Wesentlichen diesclben Verhältnisse. An ihnen scheinen manchmal schon in diesem Stadiun Abschüirungen vorzukommen. Wenigstens trifft man derartige Kernalyschnitte, welche bald durch gefarbte, bald durch blasse Fiiden untereinander in Verbindung stehen.

An selr vielen Kernen nimmt man eine oder mehrere helle Stellen von rundlicher, eckiger oder mehr länglicher Gestalt walur. Dieselben kommen sowohl an Kernen vor, welche eine nur geringe Zanahme an chromatischer Substanz erfahren haben (Fig. 6, 7 u. 8), sowie an solchen, welche von mehr oder weniger dichten Fadennetzen durchsetzt werden ( $F i g .28-36$ ) oder aber eine radiüre Aufstellung der chromatischen Elemente aufweisen (Fig. 27 u. 40). Diese hellen Felder sind zuweilen sehr klein, anderemale werden sie so gross, dass die Kerne mehr die Form von Ringen annehmen (Fig. 28, 31, 32 u. 41). Sind mehrere solche helle Stellen 
vorhanden, so entsteht das Bild, als ob die Kerne aus netzförmig verbundenen, verschlungenen oder aufgerollten Bändern zusammengesetzt wären (Fig. 36, 43, 44, 45 u. 46). Die chromatischen Fïden sind innerbalb dieser Bänder häufig, aber nicht immer kenntlich; dieselben haben dann ein mehr homogenes Aussehen. In den hellen Feldern trifft man eingebettet in eine lichte feinkörnige Substanz Körner und Fädchen, welche bald ziemlich stark, bald schwach oder gar nicht gefärbt sind; die nicht gefärbten Körner, namentlich die in der Mitte gelegenen, haben einen starken Glanz. An den Fäden lässt sich manchmal eine radiäre Anordnung nachweisen. Die Begrenzung dieser hellen Felder gegen die übrige Kernfigur ist oft eine ganz scharfe und wird zuweilen durch einen doppelten Contour bewerkstelligt (Fig. 28, 29, 30 u. 31). In anderen Fiallen erscheint die Begrenzungslinie melr zackig und wird von abtretenden blassen und gefärbten Fïden unterbrochen (Fig. 32,34 u. 35 ).

Auch bei radiärer Aufstellung der Fäden kann die Begrenzung der hellen Felder bald eine scharfe, bald eine mehr verwischte sein. Sind die in den hellen Feldern gelegenen Fiden gefürbt, so erscheinen sie als die Fortsetzung der in der ïbrigen Kernfigur enthaltenen chromatischen Elemente, möge diese nun die Form von Fäiden, Stüibchen oder Körnern besitzen. Dass dieselbe Beziehung zwischen den chromatischen Bestandtheilen der Kernfiguren und den in den hellen Feldern gelegenen nicht gefärbten Fäden besteht, ist mir zwar sehr wahrscheinlich; zu einer sicheren Ueberzeugung konnte ich aber über diesen Punkt nicht gelangen. Neben den geschilderten Formen kommen auch Kerne vor, welche bei radiärer Aufstellung der Fäden solche helle Felder nicht aufweisen (Fig. 27).

Von den auf Theilungsvorgänge an den Kernfiguren der kleineu Zellen zu beziehenden Erscheinungen soll zunächst derjenigen gedacht werden, bei welchen es möglicher Weise um einfache Abschnürungen sich handelt. Es kommen solche bei Kernen vor, welche kaum eine wesentliche Zunahme der chromatischen Substanz ïberhaupt, der chromatischen Fäden insbesondere aufweisen; aber auch Kerne, bei welchen eine solche sich eingestellt hat, die in Folge dessen von zablreichen Fäden durchsetzt werden und welche ausserdem eine mehr oder weniger intensive diffuse Färbung darbieten, zeigen Absehnitrungen der Kernfiguren bei gleich- 
zeitiger Abfurchung des Zellleibes in zwei, drei und mehr Theile. Anderemale geht ein Auftreten von hellen Feldern an einer oder mebreren Stellen in der eben geschilderten Weise voraus and dann erst kommt es zur Zerschnürung der Kernfigur. Anch an denjenigen Kernen, welche als Ringe oder Knäule von Kernbändern sich darstellen, erfolgt eine zur Theilung führende Scheidung. Ich darf in dieser Hinsicht auf meine friiheren Beobachtungen an den Zellen des Knochenmarkes verweisen. Man vergleiche ferner die Figuren 41,43,44, 45, 46 Taf. XXV u. 47 Taf. XXVI. Sebr häufig findet man Kernfiguren, welche eine bipolare oder pluripolare Anordnung. der Abschnitte darbieten, bei denen aber die an den Polen gelegenen chromatischen Gebilde durch ein, zwei, drei und mehr dunkle Fiiden unter sich in Verbindung stehen; die dazwischen gelegene Substanz ist hell und enthält blasse Fiidchen und Körneben (Fig. 48, 49 u. 50 Taf. XXVI). Auch der homogenen Kerue, welche bei netzförmiger Anordnung der einzelnen Abschnitte bereits Scheidung des Protoplasma zeigen (Fig. 56, 57 u. 58 Taf. XXVI), darf ich nicht unterlassen zu gedenken.

Dass nicht selten Kernfiguren getroffen werden, welche mit echten Mitosen in ihren verschiedenen. Stadien eine mehr oder weniger weit gehende Uebereinstimmung darbicten, verdient besonders hervorgehoben za werden.

In den grossen Zellen finden sich verbältnissmïssig sclten einfache runde Kerne; meistens sind dieselben vom Rand her mehr oder weniger tief eingeschnürt oder gelappt oder sie erseheinen als ringförmige, aufgerollte oder netzförmig verbundene Bänder; zuweilen sind die Kerne sebr stark in die Länge gezogen. Man vergleiche bezüglich der verschiedenen Formen die Figuren 59, 60, 61, 63, 65, 66, 67, 68 Taf. XXVI u. Fig. 69, 70, 71; 75, 80 und 81 Taf. XXVII, sowie die früheren Mittheilungen $\left.{ }^{1}\right)^{1.2}$ ) ïber die grossen Zellen des Knochenmarkes, mit denen sie in vielfacher. Hinsicht namentlich aber betreffs der Form vergleichbar sind.

Bei der Mebrzahl der Kerne der grossen Zellen, sie mögen eine Form haben, welche sie wollen, ist eine Zunahme der chro-

1) J. Arnold, Beobachtungen über Kerne und Kerntheilungen in den Zellen des Knochenmarkes; V irchow's Archiv Bd. 93. 1883.

2) Weitere Beobachtungen über die Theilnngsvorgänge an den Knochenmarkzellen; Virchow's Archiv Bd. 97. 1884. 
matischen Substanz nachweisbar. Die Kerne sind von kleinen rundlichen und eckigen intensiv sich färbenden Gebilden in wechselnder Zahl durchsetzt (Fig. 59, 66 Taf. XXVI u. 70 Taf. XXVII), dazwisehen liegen blasse Fäden in einer nicht zu enträthselnden Anordnung. In anderen Kernen finden sich schmälere und breitere Fäden, welche nach allen Richtungen durch die Kernsubstanz ziehen und eine netzförmige Anordnung einzuhalten scheinen. Wenigstens glaube ich mich an diesen Kernen von einer gegenseitigen Verbindung: der Fäden haben überzeugen zu können; dass aber selbst an diesem Objekt ein ganz sicherer Entscheid nicht möglich ist, lässt einen Riickschluss auf die grosse Schwierigkeit der Lösung dieser Frage zu (Fig. 60, 61, 63, Taf. XXVI u. Fig. 69, Taf. XXVII). Manche Kerne werden von einem System dunkler Fäden in sehr dichter Weise durchsetzt (Fig. 62 und 64), welche dann zuweilen eine mehr radiäre Aufstellung darbieten. Gleichzeitig kann eine mehr oder weniger starke diffuse Färbung der Kerne vorbanden sein ( $F$ ig. 60, 61, 62, 63, 64 etc.), welche manehmal in den verschiedenen Abschnitten der Kernfigur eine ungleiche ist (Fig. 68).

Die bei den kleinen Zellen in den Kernen beschriebenen hellen Felder kommen auch bei den grossen Zellen vor. Der Chromatingehalt und die Anordnung des Chromatins sind bei solchen Kernfiguren sebr verscbieden; auch die Zahl und Grösse der hellen Felder wechselt. Der in Figur 63 Taf. XXVI abgebildete Kern ist vom Rand her mehrfach eingeschnürt und schwach diffus gefärbt, dagegen von ziemlich zahlreichen Fäden durchsetzt. In seiner Mitte findet sich eine kleine scharf begrenzte helle Stelle, in deren Centrum ein glänzendes Korn, ausserdem eine lichte schwach gekörnte Substanz. Fig. 66, Taf. XXVI zeigt einen grossen am Rand stellenweise eing'ebuchteten Kern von ringförmiger Gestált, nach der einen Seite mit einem kurzen dicken Fortsatz versehen. Die Mitte der Kernfigur wird durch eine helle feinkörnige und feinfadige Substanz eingenommen. Die Form des in Fig. 69, Taf. XXVII dargestellten Kerns ist eine ähnliche; nur ist der Fortsatz nach innen gerichtet. Bei dem Kerne, welcher in Figur 70, Taf. XXVII wiedergegeben ist, finden sich zwei helle Felder, welche durch eine ziemlich breite brïckenförmige Leiste von einander getrennt werden. Manchmal sind diese Verbindungen dünner und erscheinen mehr als dickere oder feinere, heller oder dunkler gefärbte Füden (Fig. 61 u. 72), welche die Felder in versehiedenen Richtungen durchziehen. Die Form 
der Felder ist keineswegs immer eine rundliche, sondern zuweilen mehr eckige oder in die Lünge gezogene, wie bei Fig. 67; die Begrenzung derselben gegen die Kernfig'ur erseheint bald scharf, bald mehr verwischt und wird nicht selten von Fälen unterbrochen, welche man mebr oder weniger weit in die Kernfigur einerseits, die die Felder erfïllende Substanz andererseits verfolgen kann.

Bei den bisher geschilderten Kernfiguren, welche solche helle Felder umschlossen, war der Gehalt an diffusen und fadigem Chromatin ein mittlerer; es kommen dieselben aber auch an selur chromatinreichen Keruen vor. In Fig. $64 \mathrm{Taf}$. XXVI ist ein ziemlich grosser Kern abgebildet, der ausser diffusem Chromatin zahlreichc dunkle Fäden enthält und in der Mitte ein scharf begrenztes helles Feld aufweist. Auch bei diesen Formen kommt es vor, dass die hellen Felder von Strüngen durchzogen und getheilt werden (Fig. 76, Taf. XXVII).

Sind bei solchen Kernfiguren mehrere helle Felder vorhanden, so erscheinen dicselben aus netzfïmig verbundenen oder knäulförmig aufgerollten Bändern zusammengeset»t (Fig. 7\%, 74, 75, 77,81 u. 82 Taf. XXVII), die sich so intensiv färben, dass man erst nach Behandlung nit saurem Alkohol die in ihnen verlatufenden chromatischen Fiiden nachweisen kann. Bei diesen Biindern ist die Begrenzung gleichfalls bald cine scharfe und glatte, bald wird dieselbe durch dunklere und hellere Fäden unterbrochen, wolche mehr oder weniger weit in ibrem Verlauf dureh die hellen Felder sich verfolgen lassen. Anch der iussere gegen den Zellleib gerichtete Rand ist manchmal gezackt und scheinen Fiden von ihm abzutreten; ob und wie weit sie in das Protoplasma cindringen, dariber vermag ich sichere Angaben nicht zu machen.

Was die auf Theilung zu beziehenden Erscheinungen an den grossen Zellen anbelangt, so muss zuerst auf den Befund von abgeschnürten oder in Abschnürung begriffenen mit der Kernfigur noch durch Brïcken und Fäden verbundenen Kernen hingewiesen werden. Die betreffenden Kernfiguren sind zuweilen ziemlich arm an diffusem und fadigem Chromatin, anderemal zeigen sie sich nicht nur dunkler gefürbt, sondern sie enthalten chromatische Füden in grösserer Menge (Fig. 78, 79 u. 80 Taf. XXVII). Dieselben Differenzen zeigen die jungen Kerne, doch sind sie im Allgemeinen ziemlich reich an chromatischer Substanz. Diese Abschnitrungen erfolgen sehr bäufig zu verschiedenen Zeiten an der Kernfigur unter entsprechender Verkleinerung dieser; selten, wie es scheint, zertheilt 
sich die ganze Kernfigur gleichzeitig in junge Kerne. Die Abfurchung des Protoplasma vollzieht sich bald endogen, bald randständig (Fig. 79 n. 85, Taf. XXVII).

Auch an den chromatinreichen und sehr complicirten Kernfiguren, welche aus netzförmig verbundenen und knäulartig aufgerollten Bändern sich zusammensetzen, tritt die Abschniurung zuweilen gleichzeitig (Fig. 83), häufiger zu verschiedenen Perioden ein (Fig. 81, 82 a. 84, Taf. XXVII). Wenigstens trifft man sehr oft höchst complicirte Kernfiguren und neben ihnen junge Kerne, bei welchen eine brïcken- oder fadenförmige Verbindung mit der ersteren kenntlich ist, während bei anderen eine solche nicht nachgewiesen werden kann (Fig. 82). Auch bei diesen Formen erfolgt die Abschnürung der jungen Kerne keineswegs in derselben Phase; wenigstens zeigen die jungen Kerne manchmal ein sehr abweichendes Verhalten der chromatischen Substanz. Die chromatischen Fïden sind bald mehr gerüstartig oder knäulförmig angeordnet oder bieten eine mehr äquatoriale Aufstellung dar. Die Abfurchung des Protoplasma vollzieht sich auch bei diesen complicirten und chromatinreichen Figuren sowohl endogen als auch randständig.

Bei dem Versuche, die beschriebenen Formen auf die einzelnen Phasen der bekannten Kerntheilungsvorgänge zuriuckzuführen, geht man am besten von demjenigen Stadium aus, in welchem die Kerne der grossen und kleinen Zellen eine Zunahme der chromatischen Substanz darbieten. Wie oben erwähnt, wird eine solche sowohl bei den runden als auch bei den in die Länge gezogenen, gewundenen und gelappten Kernen der grossen und kleinen Zellen gefunden. Das Auftreten der chromatischen Fäden erfolgt allerdings in etwas verschiedener Weise, indem bald an dieser bald an jener Stelle, oft in unmittelbarem Anschluss an die Kernmembran intensiv sich fầrbende, rundliche, eckige and verästigte Körperchen wahrnehmbar werden, zwischen welchen zunächst blasse, später gefärbte Fäden verlaufen. Diese Umwandlungen vollziehen sich bald da, bald dort frühzeitiger, manchmal aber zur selben Zeit über den ganzen Kern hin. Auch die Zahl der chromatischen Fäden wechselt in den verschiedenen Kernen; die einen werden von einem dichten Fadensystem durchsetzt, während die anderen nur spärliche derartige Gebilde enthalten. - Dazu gesellen sich früher oder später eigenthümliche Veränderungen der Kernwandschichte, in welcher gleichfalls Fäden und Körnchen zum Vor- 
sebein kommen, bis diese scbliesslich dic Eigensehaften einer chromatischen Kernmembran darbietet. Dieselle erhält sich als solebe auch nock in einem Stadium, in welchem der Kern bereits vollständig von chromatischen Fäden durehzogen wird.

An den Kernen der kleinen Zellen ist es schwierig über die gegenseitige Lagerung und Beziehung der Fïden ins Klare zu kommen und festzustellen, ob sie nur im Verhältuiss der Contiguitit zu einander stehen oder netzartig mit einander verbunden sind. Dass man bei den grossen Kernen den Einuruck criait, als ob dic Fäden in der letzterwälnnten Weise angeordnet wären, ist oben hervorgehoben worden.

An manchen Kernen ist eine polare Orientirung der Fïlen nachweisbar, bei anderen wird eine solche vermisst. Ferner muss noch der diffusen Färbung gedacht werden, welche vicle Kerne in wechselnder Intensitait zeigen. Dass sie nur der Ausdruck einer dichtereren Lagerung der Fiden sei, wic Deny's ${ }^{1}$ ) behanptet, ist deshalb nicht wahrseheinlich, weil die Kerne bei Behandlung mit saurem Alkohol deu Farbstoff abgeben und lichter werden. Ob der Contractionszustand der Kerne anf dieses Verlatten von Einfluss sein kann, habe ich bei einer anderen Gelegenheit ausfübrlich erörtert.

Welchem der bekannten Kerntheilungsvorgäinge sind nun diese Formen beizuzählen? der echten Mitose viler demjenigen Typus, welchen ich als indirekte Fragmentirung bezeichnet habe? Beide sind charakterisirt durch eine Zuuahme der dromatischen Fäden in cinem früheren Stadium der Kerntheiluns. Wiclerholt habe ich bei der Beschreibung der indirekten Fragmentirung darauf hingewiesen, dass die Zunahme der chromatiseben Fäden, wie sie bei diesem Typus der Kerntheilung in den ersten Stadien erfolgt, in einer der echten Mitose so ähnlichen Weise sich" vollziehen könne, dass es schwierig, ja in manchen Fällen uumöglich sei zu ontseheiden, ob eine derartige Kernfigur der echten Mitose oder der indirekten Fragmentirung beizuzählen sei. Dies vorausgeschickt will ich es versuchen, auf einige Erscheinungen hinzuweisen, welche in dieser Hinsicht vielleicht verwerthbar sind.

In dieser Beziehung ist zunächst das Verhalten der geformten

1) Den ys, la cytodiérèse des cellules géantes et des petites cellules incolorès de la moelle des os. La cellule 1886. 
chromatischen Substanz zu berücksichtigen. Nach meinen Erfahrungen ist die Gestalt der chromatischen Körner, wie sie in dem ersten Stadium der indirekten Fragmentirun $g$ auftreten, verschiedenartiger und wechselnder wie bei der echten Mitose. Sie stellen sich bald als rundliche bald als eckige verschieden grosse Gebilde dar, zwischen denen blasse Füden derart verlaufen, dass die chromatischen Körner an den Knotenpunkten der letzteren zu liegen scheinen. Dazu kommt, dass das Auftreten der chromatischen Körner zu wechselnden Zeiten an den einzelnen Abschnitten des Kerns erfolgt, wodurch der Eindruck einer gewissen Ungesetzmässigkeit, in besonders auffallender Weise allerdings an den Kernen der grossen Zellen hervorgerufen wird.

Dasselbe gilt von dem Verhalten der chromatischen Fäden, welche ibberdies eine sehr verschiedene Dicke besitzen, nicht selten auffallend plump sind, mehr die Form von kurzen und dicken Stäben annehmen, welche gerade so dick als lang zu sein scheinen oder gar als rundliche Körner sich darstèllen. Ueber ähnliche Befunde hat neuerdings Flemming bei gewissen Mitosen berichtet. In wie weit unsere Befunde vergleichbar sind, wage ich vorerst nicht zu entscheiden. Bezüglich der sehr dicken und plumpen Gebilde, wie sie oben beschrieben und an manchen Figuren abgebildet sind, wird man sich ja immer die Frage vorlegen müssen, ob es sich nicht um Verklebungen mehrerer Fäden handelt.

Auch die Aufstellung der Fäden ist bei der indirekten Fragmentirung keine so regelmässige, wie bei der echten Mitose. Abgesehen davon, dass sie auch in späteren Stadien eine mehr gerüstweise oder netzförmige Anordnung zeigen, fehlt sehr bäufig eine ansgesprochene polare Orientirung. $\mathrm{Ob}$ den Fäden bei der indirekten Fragmentirung in irgend einer Phase regelmässig eine Längstheilung zukommt oder nicht, darüber kann ich keine bestimmten Angaben machen. Wäre dies nicht der Fall, so wïrle es einen weiteren wesentlichen Unterschied zwischen der echten Mitose und der indirekten Fragmentirung, welcher offenbar die beschriebenen Formen zum grösseren Theil zugeschrieben werden müssen, bezeichnen. - Auch die diffuse Färbung der Kerne liesse sich in diesem Sinne verwerthen, wenn sich herausstellte, dass sie bei der echten Mitose nicht getroffen wird.

Eine besondere Berücksichtigung verdient aber in dieser Hin- 
sicht das Verhalten der Kernmembran. Es wurde darauf hingewiesen, dass in der Kernwandschichte grläinzende Fäilen und Körner auftreten und dass diese an $\mathrm{Zahl}$ zunehmen, bis endlich die $\mathrm{Mem}$ bran die Eigenschaften einer chromatischen angenommen lat und als solche bis in spätere Phasen hinein sich erbält.

Ein weiterer wesentlicher Unterschied zwischen der typischen Mitose und der indirekten Fragmentirung wäre endlich durch das Vorkommen der Kernabschnürung sehon in diesen frühen Stadien gegeben. Wie oben mehrfach erwähnt wurde, trifft man nicht selten Kerue, welche zablreiche chromatische Fiiden in geristartiger Anordnung einschliessen, im Zustande der Theilung. Man könnte gegen diese Auffassung einwenden, dass die in Abscluniirung begriffenen Kernh:ilften schon die Stadien bis zur Knäulform in rückläufiger Reibenfolge durchgemacht hätten. Dagegen schcint allerdings das ganze Verhalten der in Abschnürung begriffenen Kernabsehnitte, insbesondere die Anwesenleit einer chromatischen Membran zu sprechen; auch der Mangel einer zwischen denselben ausgespannten achromatischen Figur ist in dieser Hinsicht $z u$ berïcksichtigen. Insbesondere lässt aber der Befund von Kernen, welche mit complicirten aus chromatinreichen Bändern bestehenden Kernfiguren nur noch durch feine Fïden verbunden sind, meines Erachtens kaum eine andere Deutung zn als die, dass auch schon in fritheren Phasen eine Abschnürung vorkomme.

Eine besondere Berücksichtigung verdicnen bei der Erörterung ter Frage, in weleher Beziehung die geschilderten Befunde zu der echten Mitose einerseits, der indirekten Fragmentirung anderseits stehen, die oben beschricbenen hellen Felder, wie sie in den einfachen und complicirten Kernfiguren der grossen und kleinen Zellen vorkommen. - Bei Gelegenheit meiner früheren Mittheilungen hatte ich auf diese Erscheinungen und deren Bedentung fiir die Erklärung der Genese der ring-, knäul- und netzförmig angeordneten Kernfiguren, wie sie im Knochenmark, in den Lymphdritisen, in der Milz und auch an anderen Stellen vorkommen, aufmerksam gemacht. Ich $\left.{ }^{1}\right)$ sprach allerdings mit einer

1) J. Arnold, Beobachtungen über Kerne und Kerutheilungen in den Zellen des Knochenmarkes, Virchow's Archiv, Bd. 90, 1883; ferner über Kerntheilung und vielkernige Zellen, Virchow's Archiv, Bd. 98, 1884 und über Theilungsvorgänge an den Wanderzellen, dieses Archiv, Bd. 30, 1887. 
gewissen Zuriicklialtung dic Vermnthung aus, dass das Auftreten dieser hellen Felder auf eine eigenthïmliche Unwwandlung der Kernsnbstanz, der Kernwandschicht insbesondere zurïckzufülıren sei, indem an zwei oder mehreren Stellen die chromatische Substanz zuriickweiche und so ring-, knäul- oder netzförmige Bänder entstehen. Dass diese bald reicher bald ärmer an chromatischen Fäden und diffus vertheilter chromatischer Substanz sind und schon sehr frühzeitig eine mebr oder weniger scharfe Begrenzung aufweisen, wurde gleichzeitig betont. Aus dem letzteren Verhalten schloss ich insbesondere auf eine Betheiligung der Kernwandschicht.

Gegen diese Mittheilungen sind verschiedene Einwände geltend gemacht worden. Da ich über den historischen Theil dieser Frage früher ${ }^{1}$ ) ausfiubrich berichtet habe, darf ich mich daranf beschränken an dieser Stelle nur des Wesentlichen zu gedenken. - Lö it $^{2}$ ), welcher tabrigens gleichfalls eine Zunahme der chromatischen Substanz bei der Fragmentirung acceptirt, ist betreffs der complicirten Kernfiguren der grossen Zellen insbesondere der Ansicht, dass sie durch Verschmelzung von Kernen immigrirter und invaginirter Zellen entstehen und meistens dem Untergang sewidmet seien, nicht der Proliferation dienen. Diese Art der Genese durfte mit Rücksicht anf die complicirte Architektur dieser Kernfiguren, namentlich aber die Zusammensetzung derselben aus Bändern, welche chromatische Fäden enthalten and von einer eigenen Kernwandschicht bekleidet werden, als sehr wenig wahrscheinlich bezeichnet werden. Ueberdies hatten bei der Beobachtung des lebenden Objektes ${ }^{3}$ ) keine Anhaltspunkte für das Vorkommen derartiger Verschmelzungen der Kerne immigrirter und invaginirter Zellen sich ergeben. Was die degenerativen Vorgünge anbelangt, so hatte $\left.\mathrm{ich}^{4}\right)$ schon vor Löwit auf diese Möylichkeit aufnerksam gemacht, zugleich aber nachgewiesen, dass auch eine fortschreitende Entwicklung an ihnen beobachtet werde. Wie begrïndet diese Anschauung war, hat die Untersuchung am

1) J. Arnold, über Theilung der Wanderzellen, dieses Archiv. Bd. $30,1887$.

2) Löwit, über Neubildung und Zerfall weisser Blutkörper. Sitzungsberichte der k. Akademie der Wissenschaften in Wien. Bd. 92, 1885.

3) J. A rn old, ïber Theilung der Wanderzellen, dieses Archiv. Bd. 30.

4) J. A raold, Beobachtungen über Kerne und Kerntheilungen in den Zellen des Knochenmarkes, Virchow's Archiv Bd. 93. 1883. 
lebenden Objekte gelchrt, bei welcher sich feststellen liess, dass bei Zellen mit solchen Kernfiguren cine Auschnürung von Kernen und Zellen sich nachwcisen lässt.

Denys, weleher eine Zunahme der chromatischen Substanz bei der Fragmentirung (Stenose), bei den in Rede stehenden Kernfiguren insbesondere, in Abrede stellt und die dunkle Fairbung lediglich auf einen Contraktionszustand der Kerne zuriickfïhrt, vertritt dic Meinung, dass die complicirten Kernfiguren durch eine Verschmelzung der sprossenförmigen Fortsätze des Kerns entstelien. Die an der Stelle der hellen Felder gelegene Sulstanz betrachtet er nicht als metamorphosirte Kernsubstanz, sondern als Zellprotoplasma. - Dass cine wirkliche Zunahme der chromatisehen Fiden bei solchen Figuren vorkommt, scheint mir zweifellos aus den friiheren, sowie den obigen Mitthcilungen hervorzugehen. Es will mir deshalb kaunı erforderlich dünken, weitere lieweise dafür beizubringen und von Neuem zu erörtern, dass und warum die Anordnung der chromatischen Fïden nicht lediglich auf einen Contractionszustand der Kerne zuritekgefiilhrt werden kann.

Mit der Mörnlickkeit, dass die complicirten Kernfignren dureh Verschmelzung der einzelnen Abschnitte des Kerns zu Stande kommen kïnnten, batte anch ich gerchnet, ja ich bin bereit dieselbe anch hente noch zuzulassev. Auf der anderen Seite muss zugegeben werden, dass sichere Beweise für das Vorkommen solcher Verschmelzungsvorgänge an diesen Kernfiguren bis jetzt nicht vorliegen. Jedenfalls wird man mit Rücksicht auf die gleich zu erïrternden Verhältnisse einräumen mitssen, nicht nur dass andere Möglichkeiten in Betracht kommen, sondern auch dass für gewisse Formen die Entstehung durch Verschmelzung sprossenförmiger Auswüchse des Kerns ausgeschlossen werden kann.

Cornil') lässt die Zellen des Knochenmarkes nur nach dem Typus der echten Mitose sich theilen. Dabei zeigen aber die Formen und Vorkommnisse, welche Cornil beschreibt, so wesentliche Abweichungen von der echten Mitose hinsichtlich der Gestalt der Kernfiguren in den einzelnen Phasen, der Reihenfolge dieser, sowie der Zeit, in welcher die Theilung an den Abschnitten der Kernfigur sich vollzieht, dass man es viel eher mit einer indirekten

1) Cornil, sur la multiplication des cellules des la moelle des os par division indirecte daus l'inflammation, Archives des Physiologie. Bd. X etc. 1887. 
Fragmentirung als mit einer typischeu Mitose zu thun zu baben glaubt. Wenn Cornil das Vorkommen der ersteren trotzdem liugnet und alle Erscheinungen anf dic letztere Kerntheilungsform zurückführen will, so muss dieses auf „Missverständniss“ beruhen. Cornil hat z. B. ganz übersehen, dass es sich bei den grossen Kerntheilungsfiguren fast niemals um einfache, sondern um mehrlache Theilungen bandelt, aber die typische Anordnumg der achromatischen und chromatischen Substanz fehlt, wie sie der echten pluripolaren Mitose zukommt. Cornil betrachtet die ring- und netzörmigen Kerne, welche wenige Fïlen enthalten als ruhende. Es scheint mir dies mit Rïcksicht anf die an ihnen beobachteten Abschnïrungsvorgïnge nicht zntreffend. Dic Herkunft und Entstehungsweise dieser Kernformen bleibt bei einer solehen Vorstellung. rïthselhaft.

An Widerspritehen herrscht somit in der Frage nach der Genese und Bedeutung der in Rede stchenden Kerufiguren kein Mangel: wic ich glaube, weil man die von mir früher bericbtete Thatsache unberiicksichtigt liess, dass die chromatinarmen und chromatinreichen derartigen Formen aus Biindern zusanmengesetzt sind, welehe eine aus Kernwandschichte bestehende Bekleidung besitzen. Der Einwurf, dass dic ehromatischen Formen nur misshandelte Mitosen und die Bünder nichts anderes scien als verklumpte chromatische Fäden, lässt eben ganz diese membranöse Bekleidung derselben ansser Acht. Ueberdies hat man iibersehen, dass es sich in allen diesen Fällen um mehrfache Theilungen handelt. Es wiirde ein zweifelloser Fortschritt scin, wenn es gelänge iiber die Entstehung dieser ans Kernwandschichte bestehenden Umhüllung der Bänder Aufschluss zu erhalten. In dieser Hinsicht verdient, wie ich glaube, die zur Bildung der oben beschriebenen hellen Felder fiihrende Metamorphose der Kernsubstanz in erster Linie beriicksichtigt zu werden. Der Befund von solchen hellen Stellen an den Kornen ist ja auch der Grund gewesen, wesshalb mir schon früher die aussebliessliche Entstehung der ring- und netzförmigen Kerne durch Verwachsung sprossenförmiger Fortsätze zweifelhaft erschien.

Wie aus den obigen Mittheilungen herrorgeht, findet man solche hellen Felder von wechselnder Grösse und in wechselnder Zahl in den Kernen und zwar sowohl in solchen mit geringem Gehalt an chromatischer Substanz, als auch an Kernen, welche 
von dichten Fadensystemen durchsetzt werden (Taf. XXV-XXVII). Ob man für diese hellen Felder eine polare Orientirung annehmen darf, ist mir fraglich; ztweilen erhielt icb den Eindruck namentlich bei der Anwesenheit nur einer, beziehungsweise zweier derartiger Stellen, als ob eine solche Anordnung bestiinde. Sind mehrere solche hellen Felder vorhanden, so ist es schwierig in dieser Beziehung eine Vorstellung, unmöglich eine Ueberzeugung sich zu versehaffen. Die Form derselben pflegt eine rundliche oder eckige zu sein; zuweilen stellen sie sich mehr als mit heller Substanz erfüllte Spalten dar, als ob es sich um eine Läng'stheilung der Kerne handelte. Die Begrenzung der Felder gegen dic eigentliche Kernfigur ist manchmal eine sehr scharfe und wird zuweilen durch einen doppelten Contour dargestellt. Anderemal erscheint diesclbe etwas unregelmässig gezackt, durch Fiilen unterbrochen und iiberhaupt weniger prïicis. An der Stelle der hellen Felder liegt eine Substanz, deren Lichtbrechnng derjenigen des Protoplasmas sehr nahe steht, manchmal vollkommen mit ibr ibereinstimmt, anderemal aber wesentlich von ihr verschicden ist. In ihr eingebettet finden sich Körner und Fädehen, welche nur selten intensiv sich farhen, meistens nur strecken- und stellenweise oder gar nicht tingirt sind; zuweilen werden die Felder von dickeren stark gefärbten Strängen durcbsetzt (Taf. XXV-XXVII). Die Anordnung der Fäden schien mir bald eine mehr netzförmige, bald eine mehr radiäre zu sein; oder aber es liess sich eine Gesetzmässigkcit in dom Verlauf der Fäden überhaupt nicht aufinden.

Diese soeben aufgezihlten Befunde sind meines Erachtens nur so zn erklïren, dass es sich um eine eigentbïmlicbe Metamorphose der Kernsubstanz handelt, bei der an gewissen Stellen das Chromatin aus den Körnchen und Fäden der Kernsubstanz, viclleicht auch aus dem Kernsaft verschwindet. Dadurch kommt es zu einer eigenthümlichen Aufhellung dieser Stellen, welche jetzt von einer lichten Substauz und blassen Körnchen und Fäden eingenommen werden. Die durch diese Metamorphose frei werdende chromatische Substanz scheint nach anderen Abschnitten der Kernfigur auszuweichen, welche jetzt aus Ringen und Knäulen chromatinreicher Bänder sich zusammensetzt. Die auf die Anwesenheit einer membranösen Bekleidung dentende Begrenzung" dieser lïsst anf eine Betbeiligung der Kernwandschicht bei diesen Vorgängen schliessen. 
Als ich vor Jahren diese hellen Felder, namentlich die kleineren Formen beobachtet, war der erste Eindruck der, dass es sich um Vacuolenbildung, somit um eine Degenerationserscheinung handle. Ich sah mich in dieser Anschauung durch die Wabrnehmung bestärkt, dass wirklich einige dieser Kerne degenerativ zu Grunde gehen. Allerdings war mir immer aufgefallen, dass in der Mitte dieser hellen Felder sebr büufig ein glänzendes Korn gelegen ist und einzelne lichte Fädchen in der Substanz der vermeintlichen Vacuole eingebettet sind, deren Abgrenzung gegen die übrige Kernsubstanz überdies als eine ganz ungewöhnliche anerkannt werden musste. Sehr bald uberzeugte ich mich aber davon, dass diese Metamorphose nicht eine Degeneration des Kerns anzeigen könne, weil das Auftreten der hellen Felder und das Verscbwinden der chromatischen Substanz an diesen Stellen von dem Auftreten einer complicirten Kernfigul begleitet bezw. gefolgt wird, deren Zusammensetzung aus mehr oder weniger chromatinreichen, mit eigener Kernwandschichte bekleideten Bändern auf eine fortschreitende Entwicklung hinwies.

Selbstverstaindlich legte ich mir schon damals die Frage vor, ob nicht dic chromatinrcichen Formen - für die chromatinarmen wäre ja eine solche Erwägung nicht zutreffend - als Mitosen aufzufassen scien, welche in Folge mangelhafter Conscrvirung diese ungewöhnliche Erscheinung darböten oder ob nicht diese Kernfiguren als nnwesentliche Abweichungen von der echten Mitose aufzufassen seien. In der ersteren Hinsicht muss ich betonen, dass diese Kernfiguren in allen Objekten, mochten sie mit ChromOsmiumessigsäure, Chrom-Ameisensäure, Chrom-Essigsäure, Platinchlorid oder Alkobol behandelt, mit Safranin, Hämatoxylin oder nach Gram gefürbt sein, in grosser Zahl vorkommen. Den Einwurf, dass wir es mit "Artefacten", „Kunstprodukten" etc. zu thun bätten, konnte ich in Anbetracht der sebr zahlreichen Versuche, welche ich mit den verschiedensten Conservirungs- und Färbungsmitteln anstellte, mir selbst nicht machen. Dass unwesentliche Abweichungen von der Mitose vorliegen, war mit Rücksicht auf die chromatinarmen Formen, namentlich aber auch in Anbetracht des Verhaltens der Kernwandschichte nicht wahrscheinlich. Immerhin lürfte es sich der Mühe lohnen, in eine etwas eingehendere Erwägung dieser Fragc einzutreten.

Machen wir den Anfang mit den einfacheren Formen, wie 
Jul. Arnold:

sie in Fig. 48, 49 u. 50 Taf. XXVI abgebildet sind und die sehr wahrscheinlich Zweitheilungen darstellen, so liegt bei diesen offenbar die Sache nicht eindentig. Von der echten Mitose weichen diese Bilder ab durch die von einer Kernhilfte zur anderen verlaufenden dunklen Fïlen. Nachdem aber von Rabl, Schottländer ${ }^{1}$ ), mil 11. A. solche verzögerte Umordnungen chromatischer Fiiden beschrieben sind, würde gegen die Deutung, dilss dies unwesentliche Aberrationen von der echten Mitose scien, keine Einwendung zu machen sein. Die in Figur 39 Taf. XXV abgebildete Kernfigur kïnnte man sich als im Stadium der äquatorialen Umordnung begriffen denken mit der Abweichung, dass die achromatische Spindelfigur sehr undentlich ist. Die in Fig. 32 Taf. XXV dargestellte Kernfigur liesse sich als Kranzform anfissen; auffallend bliebe allerdings die scharfe Begrenzung derselben regen das lichte Mittelfeld. Noch weitgehendere Uebereinstimmung bieten ja die in Fig. 24, 25, 26, 27, sowie 40,41 u. 42 Taf. XXV abgebildeten Formen dar; doch zeigen die neisten derselben mehr oder weniger wesentliche Abweichungen von der typischen Mitose bezilglich der Form und Anordnung der chromatischen Gebilde und des Verhaltens namentlich der $A$ bgrenzung der hellen Mittelfelder. Immerhin erachte ich die Anschanung, dass es sich nur un Abweichungen, vielleicht auch urn Aberrationen vou der echten Mitose handle, fïr durehaus zulaissig. Wesentlich anders liegt die Sache beziiglich der in Fig. 28-31, 35) n. 36, sowie 43-47 abgebildeten Kernfiguren. Die scharfe $A b-$ grenzung der hellen Felder (Fig. 28-31) und die Zusammensetzung der Kernfiguren aus dentlich begrenzten Bändern (Fig. 36 u. 43-47), das sind Unterschiede in der Anordnung, welche anf eine einfache Abweichung von dem Typus der echten Mitose nicht zurückgeführt werden können.

Noch melir gilt das von den Kernfiguren der grossen Zellen. Will man iiberhatpt den Versuch eines Vergleiches machen, so wïrde für die meisten der angefuihrten Kernfiguren nur dic pluripolare Mitose in Betracht kommen, da bei der Mehrzahl derselben eine mehrfache Kerntheilung: vorliegt.

Bekanntlich bestehen die echten pluripolaren Mitosen in dem Stalium der äquatorialen Umordnung, welches besonder's charakteristiseh ist, aus melrsebuenkligen chmonatischen Fignren mit

1) Schottländer 1. c., daselbst die Literatur. 
typischerAnordnung der chromatischen Fäden und den dazu gehörigen achromatischen Spindelhälften. Die in Fig. 74, 77 u. 82, Taf. XXVII dargestellten Formen haben eine gewisse Aehnlichleit mit pluripolaren Mitosen. Die dunklen Bänder könnten den Schenkeln der äquatorialen Platte verglichen werden. Hin wesentlicher Unterschied wird allerdings durch die Anordnung der Bänder, welche eine mehr netzförmige ist, durch die scharfe Begrenzung derselben (Fig. 81) und den Mangel einer achromatischen Figur bedingt; überhaupt macht der ganze Vorgang einen viel weniger gesetzmässigen Eindruck wie bei der pluripolaren Mitose. Dazu kommt, dass man chromatinarme Formen mit derselben Anordnung, allerdings noch zablreicher im Knochenmark als in der Milz trifft.

Die oben zwischen der pluripolaren Mitose und indirekten Fragmentirung angestellten Vergleiche haben zu dem Ergebniss geführt, dass zwischen beiden Vorgängen gewisse Aehnlichkeiten, andererseits aber nicht unwesentliche Abweichungen bestchen und dass diese auf die Anordnung sowohl der ehromatischen als auch der achromatischen Substanz sich beziehen.

Während bei der pluripolaren Mitose der Kernfiguren eine gesetzmässige Anordnung der chromatischen und achromatischen Faiden in den verschiedenen Phasen, namentlich aber im Stadium der äquatorialen Umordnung besteht, setzen sie sich bei der indirekten Fragmentirung ans unregelmässig gruppirten mit eigener Bekleidung ausgestatteten Bändern zusammen; an der Stelle der achromatischen Spindeln finden sich helle, von blassen Fädchen und Körnern durchsetzte Felder. Wenn meine Vermuthung richtig ist, dass bei der Entstehung dieser die Metamorphose der chromatischen Membran eine hervorragende Rolle spielt, so würde vielleicht auf das Verhalten dieser manche Abweichung in der Anordnung bei der echten Mitose und der indirekten Fragmentirung: zurückgeführt werden können, so z. B. bei der letzteren der Mangel einer achromatischen Spindel und die Anwesenheit einer Umhüllung um die die Kernfigur zusammensetzenden Gebilde, seien diese nun chromatinreich oder chromatinarm. Immerhin sind diese Differenzen, sowie die mangelnde Gesetzmässigkeit, mit welcher die einzelnen Umwandlungen sich vollziehen, so wesentlich, dass sie nicht als einfache Abweichungen von der echten Mitose aufgefasst werden können.

Noch bestimmter treten diese Unterschiede hervor in dem 
Vollzug der Abschnürung der Kerne und Zellen. Bei der bipolaren und pluripolaren Mitose erfolgt die polare Umordnung und endliche Trennung der Kerne, sowie diejenige der Zellsubstanz in ganz gesetzmässiger Weise. Allerdings hat Cornil Beobachtungen mitgetheilt, denen zufolge an einer mitotischen Kernfigur zu verschiedenen Zeiten Abschniurungen sich vollziehen sollen. Ich muss gestehen, dass sich meiner Auffassung nach ein derartiger Vorgang. nicht mit den gesetzmïssigen Erscheinungen bei der typischen pluripolaren Mitose - eine solche kann ja nur in Frage kommen in Einklang bringen liisst. Die Darstellungen, welche Cornil gibt, stimmen überdies so wenig mit dieser uberein, dass ich viel melur vermuthen möebte, es habe Cornil eine indirekte Fragmentirung vorgelegen, welche derselbe allerdings leugnet oder vielleicht richtiger gesag't nicht kennt. Dass bei dieser ähnliche Kernfiguren vorkommen, wie bei der echten Mitose, darauf wurde olsen bereits hingewiesen.

Was die Abschnürungsvorgänge bei der indirekten Fragmentirung anbelangt, so ist zunïchst hervor\%uheben, dass sie in verschiedenen Phasen der Kernumwandlung, wie es scheint, crfolgen kann. Ich habe oben berichtet, dass man an chromatinarmen Kernen grosser und kleiner Zellen und zwar an solehen sowohl mit cinfacher als auch mit complicirter Architektur Abschnirungen trifft. Es mag allerdings zweifelhaft erscheinen, ob man diese Befunde in der Weise deuten darf, dass diese Kerntheilungsvorgringe in eine sebr frithe Zeit zu verlegen seien. Man könnte sich viel mehr vorstellen, dass diese Kerne die verschiedenen Plıasen schon durchlaufen bätten und in ihrer Umwandlung in dieses Stadium eingetreten seien, ehe es zur Abschniirung kam. Dieser Auffassung ist allerdings der Befund einer membranösen Bekleidung an solchen Kernen nicht gerade günstig. Ueberdies ist zu berïcksichtigen, dass man solche Abschniirungsvorgänge an Kernen in allen Phasen der Umwandlung bei einfacher und complicirter Architektur und Struktur findet. Ich habe dieselben beobachtet bei gelappten und gewundenen Kernen mit wenigen und vielen chromatischen Fiaden, sowie bei Kernfiguren, welebe sich aus chromatinarmen und chromatinreichen Bündern zusammensetzten und wie oben bemerkt oft eine mehr oder weniger weit gehende Aehnlichkeit mit echten pluripolaren Mitosen darboten. Man vergleiche die Figuren 78, 79, 80, 81, 82 u. 84 Taf. XXVII. Dass die 
Abschnürungen der Kerne manchmal gleichzeitig, sehr hïufig aber zu verschiedenen Perioden eintreten, auch dafür finden sich auf Tafel XXVI und XXVII mehrere Beispiele. Dieselbe betreffen chromatinreiche und chromatinarme Formen.

Auch die Abschnürung des Zellprotoplasma ist eine sehr wenig gesetzmässige, bald endogen bald randständig, gleichzeitig oder zu verschiedenen Zeiten sich vollziehende. Der Unterschied in diesen Abschnürungsvorgängen der Kerne und des Protoplasma bei der echten Mitose einerseits, der indirekten Fragmentirung: andererseits liegt auf der Hand.

Der Zweck der vorstehenden Erörterungen war, auf die Uebereinstimmung und die Unterschiede zwischen der echten Mitose und der indirekten Fragmentirung hinzuweisen. Da man aus ähnlichen Ausführungen schon einmal die Schlussfolgerung gezogen hat, dass ich die mitotischen Kerntheilungsvorgänge leugne, so will ich, obgleich mir die Logik derselben nicht einleuchtet, doch nicht unterlassen zu betonen, dass ich wie für andere Gewebe überhaupt, die lymphatischen Gewebe insbesondere, so auch für die Milz das Vorkommen echter Mitosen unter normalen und pathologischen Bedingungen nicht nur nicht in Abrede stelle, sondern meines Wissens zuerst in Lymphdrtisen und Milz auf dasselbe bingewiesen habe. Ausserdem finden sich ja oben zahlreiche Angaben über Kernfiguren, welche ibrer Anordnung nach mit echten Mitosen mehr oder weniger vollständig übereinstimmen, für manche musste allerdings unentschieden gelassen werden, ob sie dieser oder der indirekten Fragmentirung beizuzählen seien. Auf eine ausführliche Darstellung der echten Mitosen einzugehen davon glaube ich absehen zu dürfen. Ich will desshalb nur erwähnen, dass ich von den charakteristischen Phasen der Mitose nur das Stadium der äquatorialen Lmordnung der chromatischen Substanz nebst den dazu gehörigen achromatischen Spindelfiguren vermisst babe. Auch typische pluripolare Mitosen beobachtete ich nur selten. Ueberhanpt sind nach meiner Erfahrung die der indirekten Fragmentirung zugehörigen Formen in der normalen Milz der. weissen Maus die überwiegenden.

In der Einleitung wurden Abweichungen und Aberrationen von der echten Mitose unterschieden und zu diesen die bomöoty. pischen und heterotypischen (Flemming), sowie die pathologischen ( $\mathrm{Rabl}$ ) Formen gerechnet. Es mag nun fraglich erscheinen, ob nicht die indirekte Fragmentirung diesen beizuzählen sei. 
564 Jul. Arnold: Ueber Kern- und Zelltheilungen in der Milz etc.

Berïcksichtigt man, dass bei dieser niemals die gesetzmässige Anordnung der chromatischen Fäden vorhanden ist, wie sie in so charakteristischer Weise der echten Mitose in den verschiedenen Stadien zukommt, dass von einer achromatischen Spindel nichts nachweisbar ist, dass die Vorgänge der Abschnürung der Kerne und Zellen in so wenig regelmässiger Weise sich vollziehen, so wird man der indirekten Fragmentirung eine Sondersteliung zuerkennen müssen. Damit soll nicht gesagt sein, dass Uebergänge zwischen allen diesen Formen der pluripolaren Mitose und der indirekten Fragmentirung insbesondere nicht existiren. Ich babe anf eine solche Möglichkeit schon an anderen Stellen ${ }^{1}$ ) hingewicsen und darf mich desbalb mit dieser kurzen Erwähnung begnügen.

Wenn auch heute schon mit grössercr Sicherheit als vor kurzem ausyesprochen werden darf, dass zahlreiche verschiedenartige und vorschiedenwerthige Abweichungen von der echten Mitose vorkommen, eine präicise Classificirung und Bezeichnung ist leider noch nicht möglich. Man könnte daran denken die indirekte Fragmentirung durcb mitotische Fragmentirung zu ersetzen, um dadurch deren Beziehung zur Mitose Ausdruck zu verleihen. Ich habe in dieser Hinsicht nur das Bedenken, dass bei der indirekten Fragmentirung die chromatischen Elemente sehr bïufig gar nicht die Gestalt von Fäden, sondern von Stübehen oder Körner haben. Eine Registrirung dieser Formen wird eben crst mïglich sein, wenn unsere Kenntnisse über Architektur, Struktur, Vorkommen, Bedeutung und Beziehung derselben zll einander dank eingehender Untersuchungen an Ausdebnung und Tiefe gewonnen haben werden.

Ich komme damit auf die im Eingang ausgesprochene Bitte zurück, dass auch Andere diesen Abweichungen von der Mitose ihre Aufmerksamkeit zuwenden and dem Studium des oben vorgeschlagenen Objektes sich unterziehen möchten.

Bezïglich der Erklärung der Abbildungen vergleiche man den Text. Sämmtliche Zellen stammen aus der Milz der weissen Maus. Die Figuren sind alle bei Zeiss $1 / 12$ h. Oc. 4 entworfen, die Einzelheiten bei stärkeren Systemen eingezeichnet.

1) J. A rnold, über Theilungsrorgänge der Wanderzellen, dieses Archiv Bd. 30, 1887. Daselbst findet sich eine ansführliche Besprechung dieser Seite der Frage. 


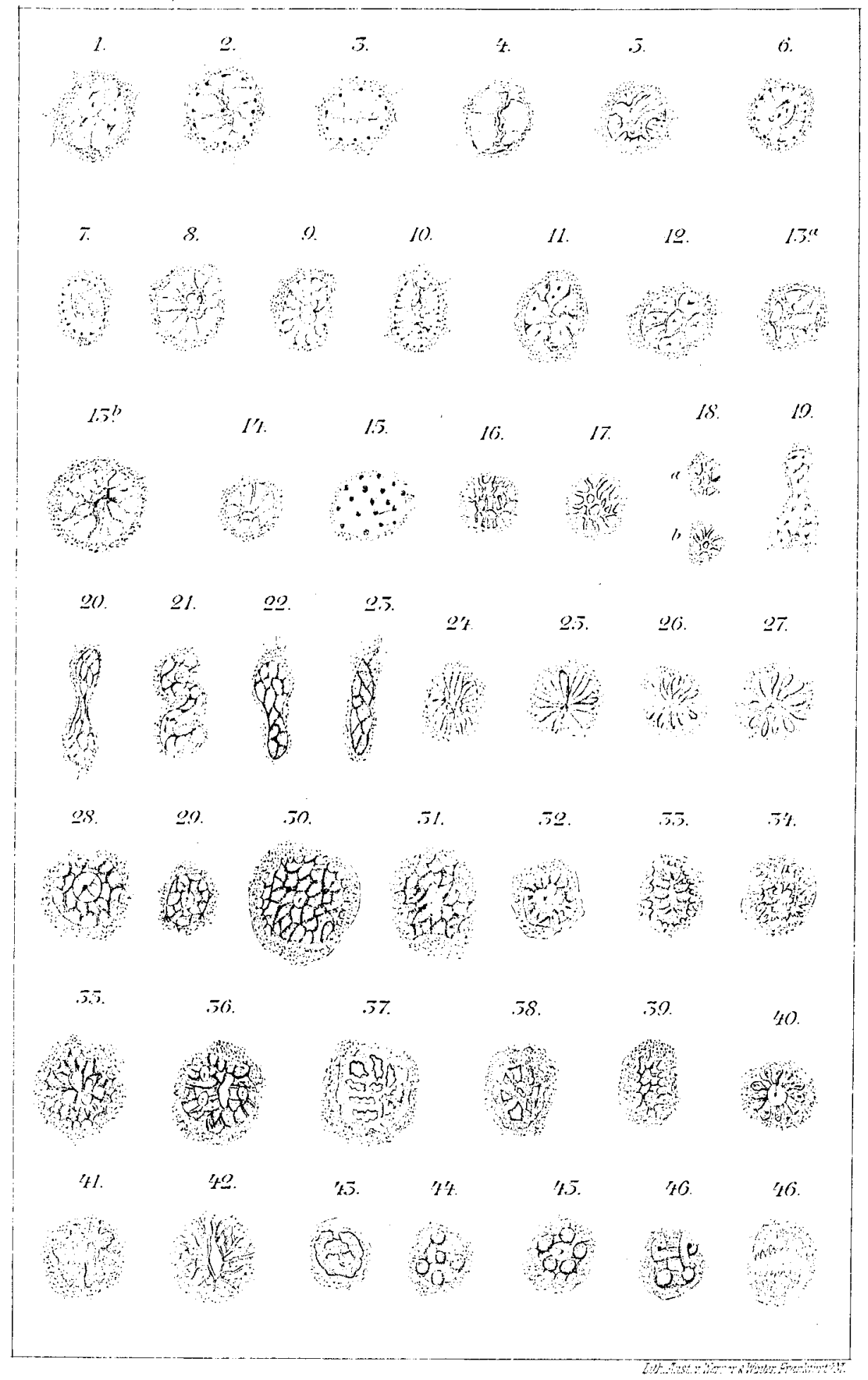




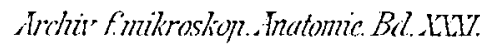

Taf.:LT7T.

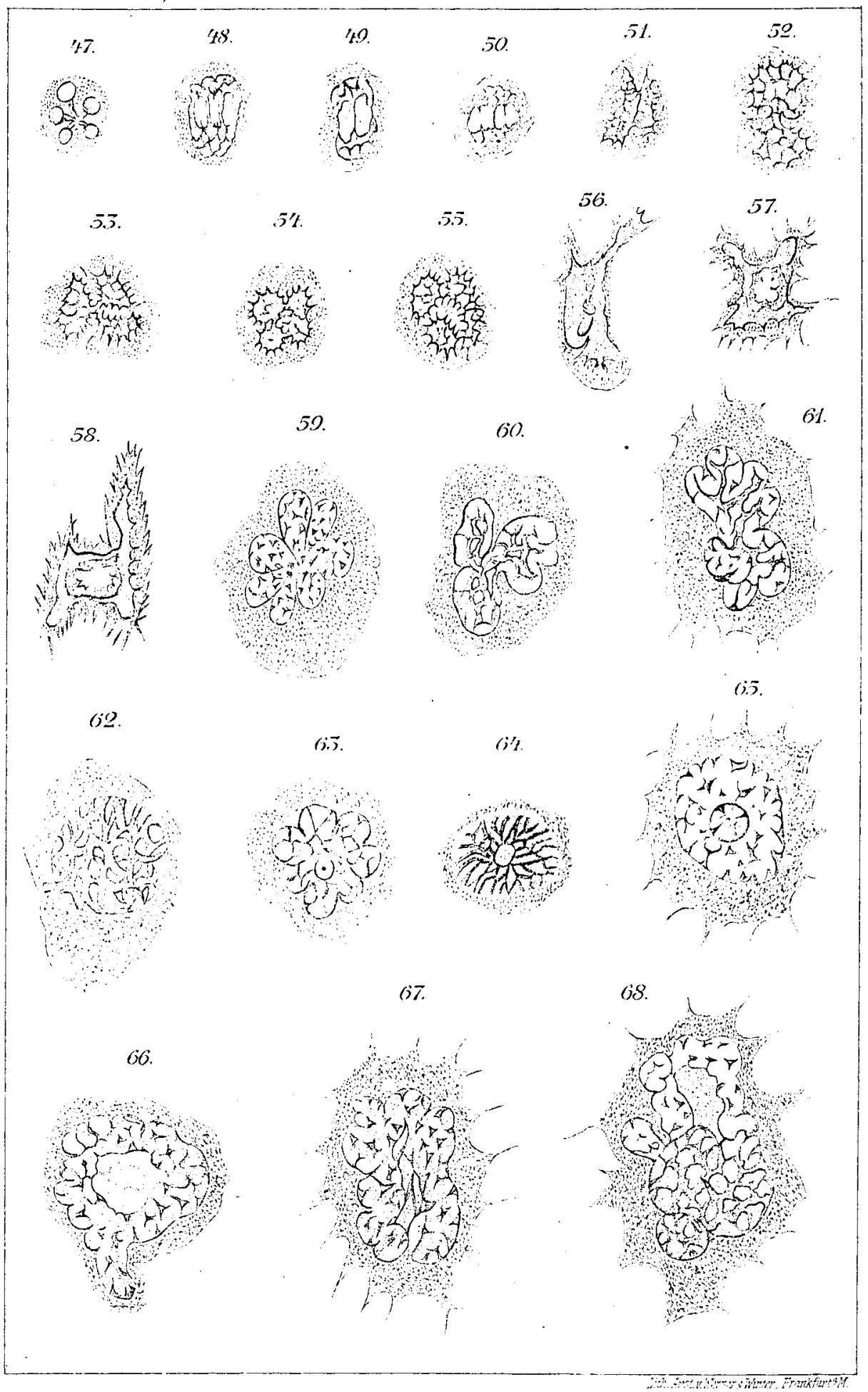




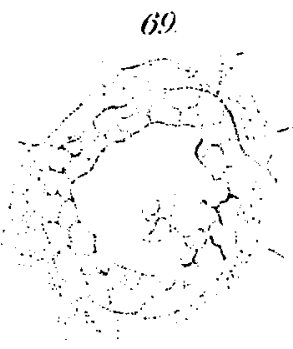

72

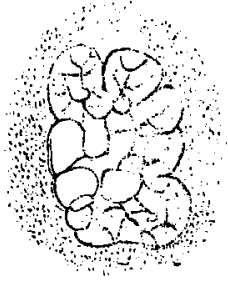

76

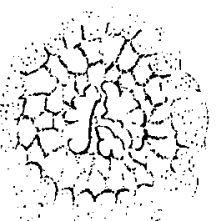

So.
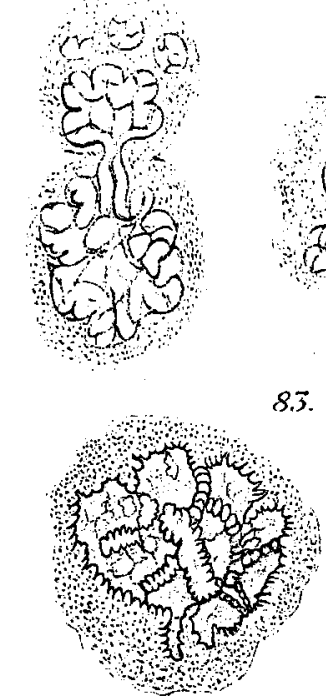

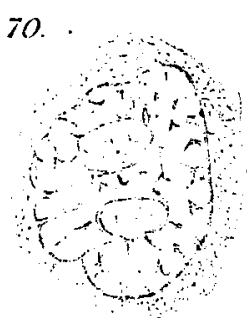

7.5.

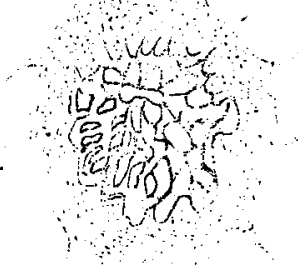

,$\cdots$

77.

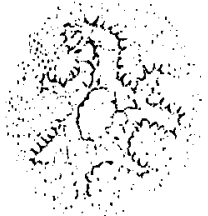

81.

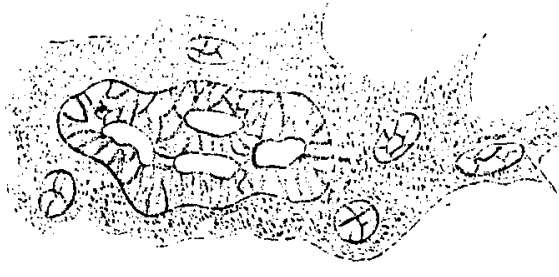

84.

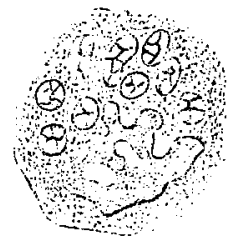

$\pi l$.

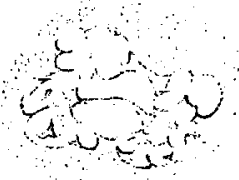

7.5.

74.
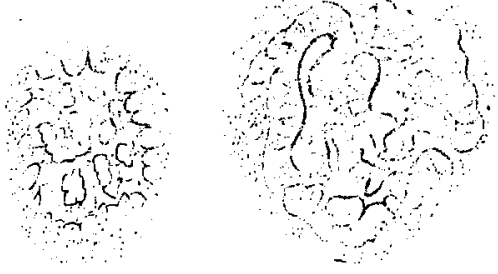

79

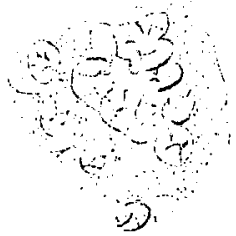

9:3.

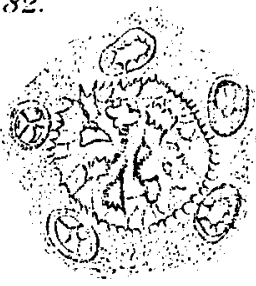

8.5.

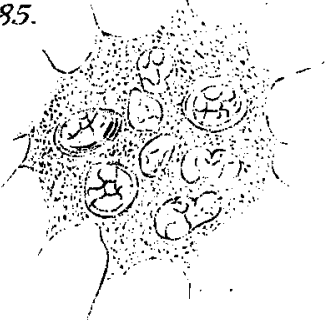

